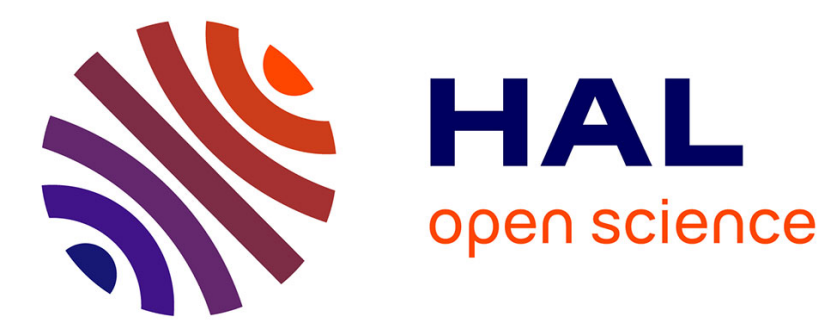

\title{
Random walks on fractal structures and percolation clusters
}

\author{
R. Rammal, G. Toulouse
}

\section{To cite this version:}

R. Rammal, G. Toulouse. Random walks on fractal structures and percolation clusters. Journal de Physique Lettres, 1983, 44 (1), pp.13-22. 10.1051/jphyslet:0198300440101300 jpa-00232136

\section{HAL Id: jpa-00232136 https://hal.science/jpa-00232136}

Submitted on 1 Jan 1983

HAL is a multi-disciplinary open access archive for the deposit and dissemination of scientific research documents, whether they are published or not. The documents may come from teaching and research institutions in France or abroad, or from public or private research centers.
L'archive ouverte pluridisciplinaire HAL, est destinée au dépôt et à la diffusion de documents scientifiques de niveau recherche, publiés ou non, émanant des établissements d'enseignement et de recherche français ou étrangers, des laboratoires publics ou privés. 
Classification

Physics Abstracts

$05.40-72.90-64.70$

\title{
Random walks on fractal structures and percolation clusters
}

\author{
R. Rammal $\left({ }^{*}\right)$ and G. Toulouse \\ Laboratoire de Physique de l'Ecole Normale Supérieure, \\ 24, rue Lhomond, 75231 Paris Cedex 05, France
}

(Reçu le 28 octobre 1982, accepté le 10 novembre 1982)

\begin{abstract}
Résumé. - La notion de dimension spectrale d'une structure self-similaire (fractale) est rappelée, et sa valeur pour la famille des tamis de Sierpinski dérivée à partir d'un argument d'échelle. On montre que diverses propriétés de marche aléatoire telles que la probabilité de marches fermées et le nombre moyen de sites visités sont gouvernés par cette dimension spectrale. On suggère que le nombre $S_{N}$ de sites distincts visités pendant une marche aléatoire de $N$ pas sur un amas infini au seuil de percolation varie asymptotiquement comme $S_{N} \sim N^{2 / 3}$, en toute dimension.

Abstract. - The notion of spectral dimensionality of a self-similar (fractal) structure is recalled, and its value for the family of Sierpinski gaskets derived via a scaling argument. Various random walk properties such as the probability of closed walks and the mean number of visited sites are shown to be governed by this spectral dimension. It is suggested that the number $S_{N}$ of distinct sites visited during an $N$-step random walk on an infinite cluster at percolation threshold varies asymptotically as : $S_{N} \sim N^{2 / 3}$, in any dimension.
\end{abstract}

1. Introduction. - It is now recognized that there are many self-similar structures (named fractals by B. Mandelbrot [1]) in nature and many ways to model them. A random walk in free space or on a periodic lattice, a linear or branched polymer, a percolation cluster at threshold are just a few well-known examples of such realizations. When considering the vast category of these self-similar geometrical spaces, it is tempting (by one turn of the crank) to regard them as basic spaces and to study some general physical effects thereon.

Among past steps in that direction, one can quote the study of random walks on percolation clusters [2] (the problem of " the ant in the labyrinth »), the study of phase transitions and transport properties on various fractals such as Sierpinski gaskets $[3,4]$, the study of the density of states on a fractal and the definition of a « fracton » dimensionality related to diffusion [5].

This paper attempts to present, in a self-contained manner, some elementary physics on selfsimilar structures. One important thread runs through the discussion : standard Euclidean spaces have translation symmetry, while self-similar spaces have dilation symmetry. As a consequence, whereas Euclidean spaces are well characterized by one space dimension $: d$, self-similar spaces require the definition of (at least) three dimensions $: d$, the dimension of the embedding Euclidean

(*) Permanent address : CNRS-CRTBT, B.P. 166, 38042 Grenoble, France. 
space; $\bar{d}$, the fractal (Hausdorff) dimension [1]; $\tilde{d}$, the spectral (fracton) dimension [5]. For Euclidean spaces, these three dimensions are equal. This can be construed as an " accidental " degeneracy and it is of interest to sort out the different qualifications of the dimensionality, in some of the many formulae where it enters. In this sense, the study of physics on self-similar spaces is not only an exploration of a new world, it also allows for a deeper understanding of the physics on more traditionally trodden spaces.

2. The spectral dimensionality $\tilde{\boldsymbol{d}}$. - On a periodic Euclidian lattice, of dimensionality $d$, the density of states $\rho(\omega)$, for excitations of frequency $\omega$, can be derived from a knowledge of the dispersion relation $\omega(k)$, where $k$ is a wave-number, and from a counting in $k$-space (reciprocal space) :

$$
\rho(\omega) \mathrm{d} \omega=\frac{\mathrm{d}^{d} k}{(2 \pi)^{d}}
$$

Therefore the argument hinges on the translation invariance of the lattice, via a Fourier space analysis of the modes and the definition of a wave-number.

In the vicinity of the lower edge of the spectrum (e.g. for elastic vibrations), the dispersion relation becomes a simple power law :

$$
\omega \sim k,
$$

and the low-frequency density of states also :

$$
\rho(\omega) \sim \omega^{d-1} .
$$

It turns out that a simple law behaviour may also be found on self-similar structures. In this case, the existence of the power law stems from a scaling argument, which is more general and deeper than the previous one, restricted to translation-invariant media.

2.1 SCAling ARgument. - To be specific, let us consider an elastic structure consisting of $N$ identical units of mass $m$, linked with springs of strength $K$. The microscopic frequency scale is then set by $\omega_{0}^{2}=K / m$. If the structure is self-similar, $N$ is related to $L$, the length scale of the structure by

$$
N(L) \sim L^{\bar{d}}
$$

This relation defines the fractal dimensionality $\bar{d}$ of the structure.

It implies that, under a scale transformation by a factor $b$, the number $N$ varies according to

$$
N(b L)=b^{\bar{d}} N(L),
$$

and correspondingly the density of states (per elementary unit) scales according to :

$$
\rho_{L}(\omega)=b^{\bar{d}} \rho_{L / b}(\omega)
$$

If, under this scale transformation, the mode frequency has also a scaling behaviour :

$$
\omega(L / b)=b^{a} \omega(L),
$$

it follows that :

$$
\rho_{L / b}(\omega)=b^{-a} \rho_{L}\left(\omega b^{-a}\right)
$$


and using (6) :

$$
\rho_{L}(\omega)=b^{\bar{d}-a} \rho_{L}\left(\omega b^{-a}\right) .
$$

Taking $b=\omega^{1 / a}$ in the r.h.s., one gets a power law :

$$
\rho(\omega) \sim \omega^{\bar{d} / a-1},
$$

from which one defines the spectral dimensionality $\tilde{d}$ as $\tilde{d}=\bar{d} / a$.

On regular Euclidean lattices, (5) holds with $\bar{d}=d$, the Euclidean dimension, and (2) implies (7) with $a=1$. Thus the spectral dimension $\tilde{d}$ is equal to $d,(10)$ reducing to (3), as it should.

In order to prove that a scaling relation like (7) may indeed hold on self-similar structures, an example is given below.

2.2 EXAMPlE : SieRPINSKI GASKETS. - The Sierpinski gaskets form a family of self-similar structures, which can be built in any Euclidean dimension (see Fig. 1, for $d=2$ ) and which have been already elicited for studies [3-5], because they lend themselves particularly conveniently to scaling approaches. Their fractal dimension is easily found : $\bar{d}=\frac{\ln (d+1)}{\ln 2}$.

Starting from the equations of motion for elastic vibrations (one equation for each site), one eliminates the amplitudes corresponding to the sites located at mid-points of triangle edges at the lowest scale. This decimation procedure leads to a reduced set of equations, describing the same physics on a gasket scaled (down) by a factor $b=2$. It turns out that the decimation introduces no new coupling constants and leads to a simple renormalization of the frequency (appendix). Thus the ratio $\omega^{2} / \omega_{0}^{2}$ is simply replaced by $\frac{\omega^{\prime 2}}{\omega_{0}^{2}}=\frac{\omega^{2}}{\omega_{0}^{2}}\left(d+3-\frac{\omega^{2}}{\omega_{0}^{2}}\right)$.

In the notations of equation(7),

$$
\omega=\omega(L), \quad \omega^{\prime}=\omega\left(\frac{L}{2}\right),
$$

and at low frequency $\omega / \omega_{0} \ll 1$, one obtains

$$
\omega^{2}\left(\frac{L}{2}\right)=(d+3) \omega^{2}(L),
$$

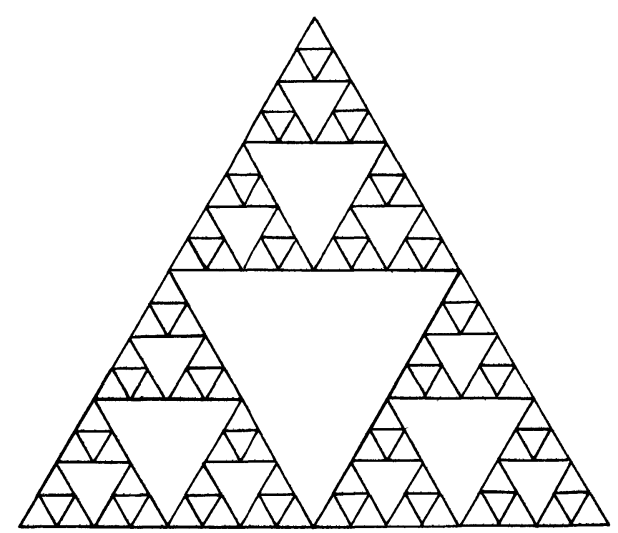

Fig. 1. - The two-dimensional $(d=2)$ Sierpinski gasket. The iteration process making the structure selfsimilar should be pursued indefinitely. The figure shows the structure after four stages of iteration from the lowest scale (elementary triangle). 
a relation which is cast in the form of equation (7) with :

$$
2^{a}=(d+3)^{1 / 2}
$$

Thus, exponent $a$ is obtained :

$$
a=\frac{\ln (d+3)}{2 \ln 2}
$$

and the spectral dimension of a Sierpinski gasket in dimension $d$ is :

$$
\tilde{d}=2 \frac{\ln (d+1)}{\ln (d+3)} \text {. }
$$

This result may be derived differently. We have chosen this derivation for its simplicity.

The density of states on a gasket is actually a highly singular function of frequency [6] and the low frequency behaviour described by equation (10) is a smoothed expression, to be used in integrals, such as those involved in the computation of thermodynamic quantities at low temperatures.

2.3 INEQUALITIES BETWEEN DIMENSIONS. - It is easy to check that the three dimensions $(d, \bar{d}, \tilde{d})$, calculated above for the family of Sierpinski gaskets, are ordered as follows :

$$
d \geqslant \bar{d} \geqslant \tilde{d} .
$$

The equalities hold for $d=1$, which is the baby of the family. When $d$ increases, $\bar{d}$ grows monotonically and is unbounded, whereas $\tilde{d}$ approaches its upper bound $\tilde{d}=2$.

The double inequality (14) appears to hold generally (there is no counter-example that we know of). It would be useful, for various reasons which will become clearer later, to know a family of fractals, as simple as the gaskets, where $\tilde{d}$ would not be restricted to values smaller than 2 .

3. Random walks. - Classical diffusion and random walks in Euclidean spaces are a well established field of physics. We direct the reader to a recent review [7] for references.

Several properties will be examined, starting with the fundamental one : the mean-squared displacement from the origin at time $t$ (or after $N$ steps on a network). An exponent $v$ is defined from the asymptotic behaviour :

$$
\left\langle R^{2}\right\rangle \sim t^{2 v}, \quad t \text { large. }
$$

In a Euclidean space, of dimension $d$, it is well known that :

$$
v=\frac{1}{2}
$$

independent of the dimensionality. Indeed, the law (15) is obtained as solution of a diffusion equation, which is analogous to the wave equation for vibrations, except for the replacement of a second by a first time derivative. Therefore, for simple dimensional reasons, the counterpart of equation (2) is equation (15) with the value (16) for the exponent.

From the arguments developed in section 2, if on a fractal structure equation (7) holds, then it follows similarly that :

$$
v=\frac{1}{2 a}=\frac{\tilde{d}}{2 \bar{d}}
$$

As it should, the standard value $\frac{1}{2}$ is recovered on an Euclidean lattice, where $\tilde{d}=\bar{d}$. 
3.1 Self-AVOIDING WALKS. - Expression (17) implies that the fractal dimensionality of a random walk (on a fractal structure) is $\bar{d}_{R}=\frac{1}{v}=2 \frac{\bar{d}}{\tilde{d}}$ Therefore two such walks will generically cross each other (or one walk will self-cross) if and only if $2 \bar{d}_{R} \geqslant \bar{d}$. This condition, which reduce to $\widetilde{d} \leqslant 4$, determines when the excluded volume restriction, in a self-avoiding walk, is relevant. It is worth noting that this condition depends only on the spectral dimension.

3.2 Closed WALKS AND SCALING LAwS. - The probability of return to the origin after $N$ steps, i.e. the probability of closed walks of length $N$, is called $P_{0}$. On a Euclidean lattice, it is easy to show [8] that :

$$
P_{0} \sim \frac{1}{R^{d}} \sim \frac{1}{N^{d / 2}}
$$

On fractals, such as the Sierpinski gaskets, for which (17) holds, the generalization of (18) is :

$$
P_{0} \sim \frac{1}{R^{\bar{d}}} \sim \frac{1}{N^{\tilde{d} / 2}}
$$

More generally, it is possible to define a set of critical exponents, in analogy with the Gaussian model of phase transitions [8]. Thus exponent $\gamma$ is defined from the total number of random walks of length $N$ and found equal to 1, for random walks on fractals as well as on Euclidean lattices. The other exponents can be derived from $v$ and $\gamma$, using the standard scaling laws of the theory of phase transitions. It is interesting to note that one of these scaling laws, the Josephson scaling law :

$$
d v=2-\alpha,
$$

involves explicitly a space dimensionality. On a fractal structure, it is the fractal dimensionality $\bar{d}$ which enters (20). This choice is consistent with (19) since the general expression for $P_{0}$ is [8] :

$$
P_{0} \sim \frac{1}{N^{\gamma+1-\alpha}}
$$

3.3 NUMBER OF VISITED SITES. - The average number $S_{N}$ of distinct sites visited during an $N$-step random walk is a quantity which has been studied for thirthy years [7] on Euclidean lattices and which is of interest for various problems (diffusion in the presence of traps, for instance).

It will be helpful to recall some established results for the asymptotic behaviour of $S_{N}$ on Euclidean lattices :

$$
\begin{aligned}
S_{N} & \sim\left(\frac{8 N}{\pi}\right)^{1 / 2}+\cdots, & d & =1, \\
& \sim \frac{\pi N}{\ln N}+\cdots, & d & =2, \\
& \sim C N+C^{\prime} N^{2-d / 2}+\cdots, & 2<d & <4, \\
& \sim C N+C^{\prime} \ln N+\cdots, & d & =4,
\end{aligned}
$$

where $C, C^{\prime}$ denote lattice-dependent numerical factors.

The escape probability $E$, i.e. the probability that the walker never returns to the origin, is

$$
E=\lim _{N \rightarrow \infty} \frac{S_{N}}{N}
$$


The probability of eventual return to the origin, $F=1-E$, is equal to unity for $d \leqslant 2$, whereas $0<F<1$ for $d>2$.

A question arises : which dimension governs the behaviour of $S_{N}$ on a fractal lattice ? We argue that it is the spectral dimension $\tilde{d}$.

Explicitly, we claim that :

$$
\begin{array}{ll}
S_{N} \sim N^{\tilde{d} / 2}, & \tilde{d}<2, \\
S_{N} \sim N, & \tilde{d}>2 .
\end{array}
$$

For Euclidean lattices, $\tilde{d}=d$, and (24) is consistent with (22). Let us introduce the notion of the number of accessible sites $\Sigma_{N}$, in a random walk of $N$ steps. The sites, which are effectively accessible, are those located within a radius $R_{N}$ of the origin :

$$
\Sigma_{N} \sim R_{N}^{\bar{d}} \sim N^{\tilde{d} / 2} .
$$

Note that $P_{0}$, the probability of return to the origin after $N$ steps, given in (19), can be recast as $P_{0} \sim\left(\Sigma_{N}\right)^{-1}$, which says that the walker may find itself, after $N$ steps, anywhere within the accessible cloud.

The number of visited sites $S_{N}$ cannot exceed either the number of accessible sites $\Sigma_{N}$ or the number of steps $N$. Formula (24) simply states that the asymptotic behaviour of $S_{N}$ is governed by the lower of these two upper bounds. It appears as the most natural generalization of formula (23). We have proved its validity for Sierpinski gaskets, using a decimation procedure analogous to that of the appendix for the generating function [7] of the walk.

3.4 Localization.-For Euclidean lattices, P. B. Allen [9] has discovered an interesting analogy between classical diffusion and quantum localization. Transposing his results to fractal lattices and using (24), one reaches the prediction that the $\beta$-function defined in [10],

$$
\beta(g)=\frac{\mathrm{d} \ln g}{\mathrm{~d} \ln L},
$$

where $g(L)$ is the conductance of a sample of size $L$ should tend, for $g$ large, toward the limit value :

$$
\beta_{L}=\frac{\bar{d}}{\bar{d}}(\tilde{d}-2)
$$

But the conductance of a Sierpinski gasket of size $L$ is easy to calculate [3] and found to scale as :

$$
g(L) \sim L^{\beta_{L}},
$$

where $\beta_{L}$ has precisely the value predicted in (27). This lends further support to the idea that formulae (24) and (27) might hold generally. For fractals with $\tilde{d}>2$, a mobility edge, for electrons in a random potential, is predicted to occur with a localization length exponent $v_{L}=\left(\beta_{L}\right)^{-1}$.

4. Application to the percolation problem. - The percolation problem is also a well established field of physics and we direct the reader to [11] for references.

At a percolation threshold, the infinite cluster is a fractal object. Therefore, the concepts of the previous sections can be brought to bear on the physics in the vicinity of a percolation threshold and this seems, at the present time, to be perhaps the major application.

It has been early recognized that the fractal dimension of a percolating cluster at threshold can 
be expressed in terms of the Euclidean dimension $d$ and of critical exponents of the percolation transition $\left(\beta_{\mathrm{p}}, v_{\mathrm{p}}\right.$, indexed by $\mathrm{p}$ for percolation, are defined below in equations (30) and (34)) :

$$
\bar{d}=d-\frac{\beta_{\mathrm{p}}}{v_{\mathrm{p}}} .
$$

In order to calculate the spectral dimension $\tilde{d}$, we shall use a simple crossover argument. Slightly above the percolation threshold $(\Delta p>0)$, there is one dominant length scale, the correlation length $\xi_{\mathrm{p}}$ :

$$
\xi_{\mathrm{p}} \sim \frac{1}{(\Delta p)^{v_{\mathrm{p}}}}
$$

For excursions smaller than $\xi_{\mathrm{p}}$, the diffusion law is expected to be of the form of equation (15) whereas, for larger excursions, a standard power law is recovered :

$$
\left\langle R^{2}\right\rangle \sim D t,
$$

The Einstein relation between the conductivity $\sigma(p)$ and the diffusion constant $D(p)$ can be written as :

$$
\sigma(p)=P(p) D(p),
$$

where $P(p)$ is the infinite cluster density. In the vicinity of the percolation threshold :

$$
\sigma(p) \sim(\Delta p)^{t},
$$

(the exponent $t$ should not be confused with time) and

$$
P(p) \sim(\Delta p)^{\beta_{\mathrm{p}}},
$$

implying :

$$
D(p) \sim(\Delta p)^{t-\beta_{\mathrm{p}}} .
$$

Expressing the equivalence of equations (15) and (31) for $R \sim \xi_{\mathrm{p}}$, we obtain :

$$
(\Delta p)^{-2 v_{\mathrm{p}}} \sim \tau^{2 v} \sim \tau(\Delta p)^{t-\beta_{\mathrm{p}}}
$$

which allows for a determination of the random walk exponent $v$ :

$$
v=\frac{v_{\mathrm{p}}}{t-\beta_{\mathrm{p}}+2 v_{\mathrm{p}}},
$$

and of a characteristic time scale :

$$
\tau(p) \sim(\Delta p)^{\beta_{\mathrm{p}}-t-2 v_{\mathrm{p}}} .
$$

The spectral dimensionality $d$ of the percolating cluster is then derived from equations (17) and (37) as :

$$
\widetilde{d}=2 \frac{\mathrm{d} v_{\mathrm{p}}-\beta_{\mathrm{p}}}{t-\beta_{\mathrm{p}}+2 v_{\mathrm{p}}} .
$$

This result, already present in the work of E. Shender [12], has been discussed in its generality by S. Alexander and R. Orbach [5]. In addition, they have made the interesting observation that $\tilde{d}$, as determined by (39), seems to be close to $\frac{4}{3}$, for percolation in any Euclidean dimension $d, 1<d \leqslant 6$. 
Now formula (24), plus the observation $\tilde{d}=\frac{4}{3}$, lead to a simple universal prediction

$$
S_{N} \sim N^{2 / 3}
$$

for the mean number of distinct sites visited during an $N$-step random walk on a percolating cluster, in any dimension.

Such a remarkable prediction, which lends itself particularly well to numerical check, calls for a physical explanation which we now present.

Consider the quantity $\mathrm{d} S_{N} / \mathrm{d} N$ which is the probability of access to a fresh site, after $N$ steps. The claim is that, on a percolating cluster which is just marginally infinite, this probability of escape is of the order of the relative fluctuation in the number of accessible sites, due to the random nature of the percolation problem itself :

$$
\frac{\mathrm{d} S_{N}}{\mathrm{~d} N} \sim \frac{\sqrt{\Sigma_{N}}}{\Sigma_{N}} \sim \frac{1}{\sqrt{S_{N}}}, \quad \text { using } \quad \Sigma_{N} \sim S_{N} ;
$$

(41) implies (40). Another way of phrasing the same argument consists in defining the " open frontier " of the random walk as the product of the probability of access to a fresh site $\mathrm{d} S_{N} / \mathrm{d} N$, by the number of accessible sites after $N$ steps $\Sigma_{N}$. It is then argued that this open frontier is marginally equal to the fluctuation in the number of accessible sites, due to the random percolation process.

Clearly, this is physical intuition, not a demonstration. Is it exact or just a, somewhat accidentally, very good prediction, like the Flory value for the polymer exponents ? Here is a valuable challenge for future study, since (40) via (39) implies a relation (the existence of which has been long questioned) between dynamic $(t)$ and static $\left(\beta_{\mathrm{p}}, v_{\mathrm{p}}\right)$ percolation exponents.

Finally, the situation above the upper critical dimension $d_{\mathrm{c}}=6$ for percolation requires discussion. For $d \geqslant 6$, it is generally accepted that $\beta_{\mathrm{p}}=1, v_{\mathrm{p}}=\frac{1}{2}, t=3$, independent of $d$. Therefore the random walk exponent $v$ is expected to stick to the value $v=\frac{1}{6}$, according to equation (37). However it remains an open question whether the dimensions $\bar{d}$ and $\tilde{d}$ are constant or linear functions of $d$, for $d>6$.

Coming now to transport properties on the percolating cluster, we firstly recognize that the value of $\beta_{L}$ given in (27) can be recast, using (39), as

$$
\beta_{L}=d-2-\frac{t}{v_{\mathrm{p}}} .
$$

Since $\beta_{L}$ permits a fine measure of the degree of localisation, it is interesting to compare its value for the percolating clusters and for the Sierpinski gaskets, as a function of the embedding Euclidean dimensionality. This is shown on figure 2 .

Whilst, for Sierpinski gaskets, $\beta_{L}$ increases monotonically with $d$, figure 2 shows that, for percolating clusters, it is always smaller (except for $d=1$ ) and furthermore it varies essentially in the opposite direction, $\beta_{L}$ being a decreasing function of $d$, for $2<d<6$. This demonstrates clearly that the Sierpinski gaskets cannot be accepted as realistic quantitative models for percolating clusters; instead, their usefulness lies at a qualitative level. The fact that $\beta_{L}$, for percolating clusters, remains always far from its critical value $\beta_{L}=0$ suggests that the localization in a random potential will be always strong, an observation which is consistent with recent results [13] suggesting that the threshold for appearance of extended states, in the quantum percolation problem, lies not at $p_{\mathrm{c}}$, the percolation threshold, but higher.

5. Conclusion. - The main content of this paper is expressed in our equation (40), which sheds a new light on the old percolation problem. We have presented evidence that this simple universal law is close to the truth. Further study is necessary to decide whether it is exact or not. At stake is the existence of a relation between dynamic and static percolation exponents. 


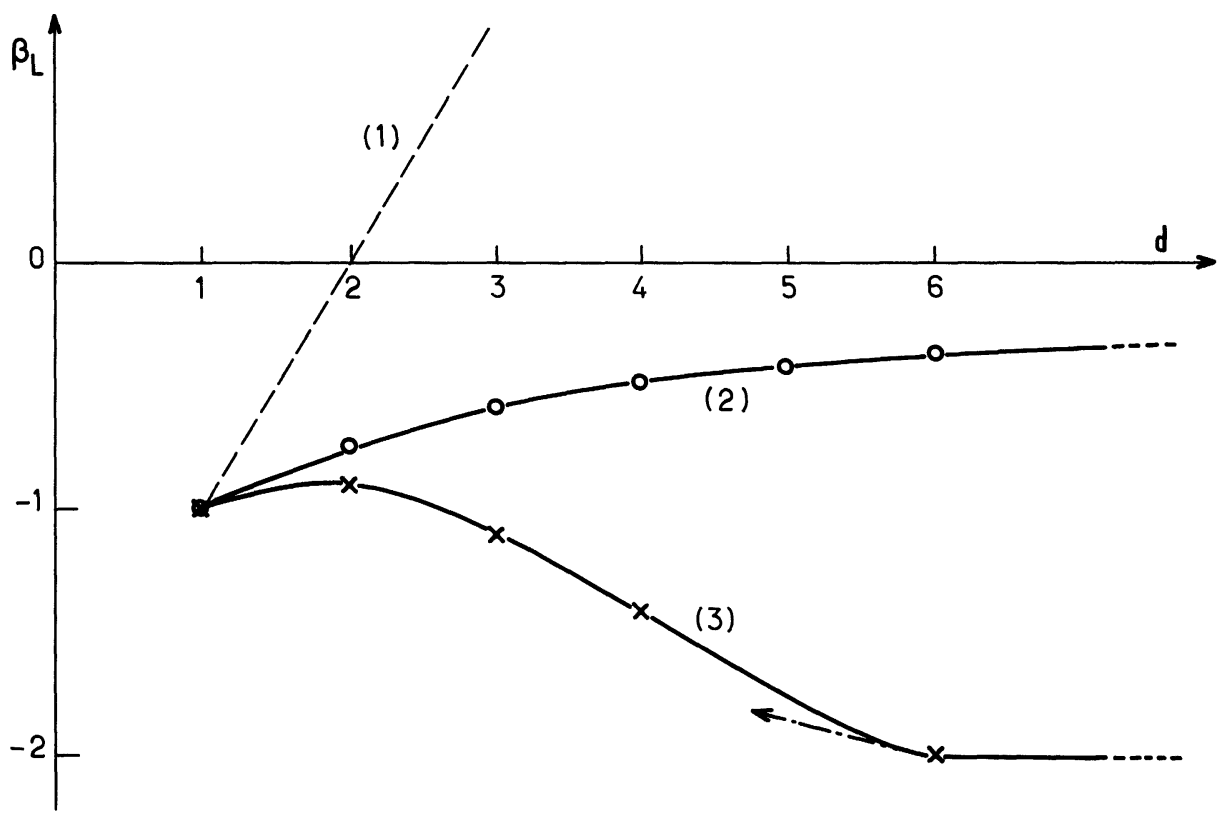

Fig. 2. - The exponent $\beta_{L}$, defined in equation (28), characterizes the size dependence of the conductance. It is plotted here as a function of the Euclidean dimension $d$ for three families of structures. Curve(1), dashed : Euclidean lattice. Curve (2) : Sierpinski gaskets. Curve (3) : percolating clusters. For this last curve, the tangent at $d=6$ can be obtained from an $\varepsilon=6-d$ expansion : $\beta_{L}=-2+\frac{5}{21} \varepsilon$ [using equation (42) and reference [11]].

Acknowledgments. - Discussions with R. Orbach and J. Vannimenus are gratefully acknowledged.

Appendix : Recursion equations for lattice vibrations on a Sierpinski gasket. - We derive first the recursion equations for the two-dimensional $(d=2)$ Sierpinski gasket (Fig. 1). Identical masses $m$ are located at the sites of the lattice, joined by springs of strength $K$. Let us denote

$$
\lambda=\frac{m \omega^{2}}{K}=\frac{\omega^{2}}{\omega_{0}^{2}}
$$
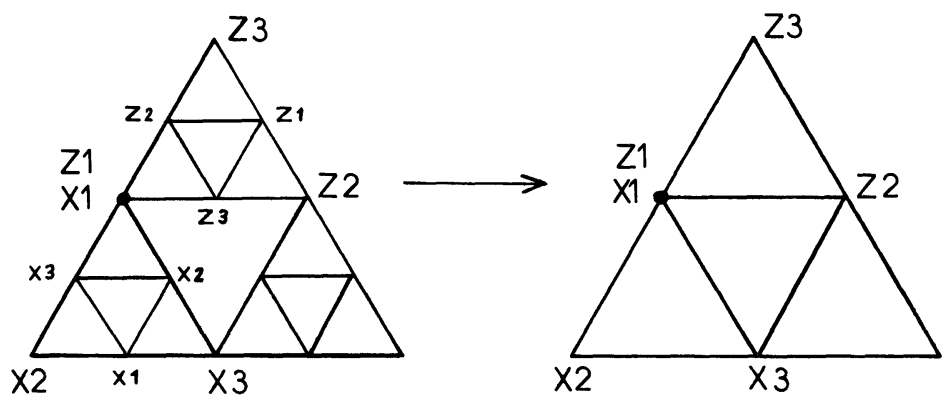

Fig. 3. - Principle of the decimation procedure, used in the appendix, to derive the recursion relations for elastic vibrations on a two-dimensional Sierpinski gasket. 
the reduced squared frequency and $\left\{X j \mathrm{e}^{i \omega t}\right\}$ the eigenstates associated with a mode of frequency $\omega$.

The set of equations of motion for the mid-points of the lower left triangle (in Fig. 3) is :

$$
\begin{aligned}
& \lambda x_{1}=\sum_{\delta}\left(x_{1}-x_{\delta}\right) \\
& \lambda x_{2}=\sum_{\delta}\left(x_{2}-x_{\delta}\right) \\
& \lambda x_{3}=\sum_{\delta}\left(x_{3}-x_{\delta}\right)
\end{aligned}
$$

where $\delta$ denotes a neighbouring site of $x_{1}$ (resp. $x_{2}, x_{3}$ ).

The corresponding equation for $X_{1}\left(\right.$ or $\left.Z_{1}\right)$ is :

$$
\lambda X_{1}=4 X_{1}-\left(x_{2}+x_{3}+z_{2}+z_{3}\right)
$$

Using (A.1), one extracts $x_{1}, x_{2}, x_{3}$ as functions of $\left\{X_{i}\right\}$ and the same for $\left\{z_{i}\right\}$ as functions of $\left\{Z_{i}\right\}$. Inserting these values in (A.2), one obtains a new equation where $X_{1}$ is a function of $X_{2}, X_{3}$ and $Z_{2}, Z_{3}$. This relation can be cast in the form of the first equation (A.1), with the renormalization :

$$
\lambda \rightarrow \lambda^{\prime}=\lambda(5-\lambda)
$$

For general value of the dimension $d$, one obtains after a rather cumbersome algebra the simple generalization :

$$
\lambda^{\prime}=\lambda(d+3-\lambda) .
$$

\section{References}

[1] Mandelbrot, B. B., Fractals : Form, Chance and Dimension (Freeman, San Francisco) 1977.

[2] De Gennes, P. G., La Recherche 7 (1976) 919 ;

Mitescu, C. D., Ottavi, H., RoussenQ, J., AIP Conf. Proc. 40 (1979) 377 ;

Straley, J. P., J. Phys. C 13 (1980) 2991.

[3] Gefen, Y., Aharony, A., Mandelbrot, B. B., Kirkpatrick, S., Phys. Rev. Lett. 47 (1981) 1771.

[4] Rammal, R., Toulouse, G., Phys. Rev. Lett. 49 (1982) 1194.

[5] AleXander, S., Orbach, R., J. Physique Lett. 43 (1982) L-625.

[6] Rammal, R., to be published.

[7] Montroll, E. W., West, B. J., in Fluctuation Phenomena, Eds. E. W. Montroll, J. L. Lebowitz (NorthHolland) 1979.

[8] See for instance Toulouse, G. and PfeUty, P., Introduction au groupe de renormalisation (PUG) 1975.

[9] Allen, P. B., J. Phys. C 13 (1980) L-667.

[10] Abrahams, E., Anderson, P. W., Licciardello, D. C. and Ramakrishnan, T. V., Phys. Rev. Lett. 42 (1979) 673.

[11] Stauffer, D., Phys. Reports 54 (1979) 1.

[12] Shender, E. F., J. Phys. C 9 (1976) L-309.

[13] Shapir, Y., Aharony, A. and Harris, A. B., Phys. Rev. Lett. 49 (1982) 486. 